\title{
Effectiveness and adverse events of topical and allergen immunotherapy for atopic dermatitis: a systematic review and network meta-analysis protocol
}

Luis Guillermo Gómez-Escobar', Hansel Mora-Ochoa', Andrea Vargas Villanueva', Loukia Spineli², Gloria Sanclemente ${ }^{3}$, Rachel Couban ${ }^{4}$, Elizabeth García ${ }^{5}$, Edgardo Chapman ${ }^{5}$ and Juan José Yepes-Nuñez ${ }^{1,5^{*}}$ (D)

\begin{abstract}
Background: Atopic dermatitis (AD) is an inflammatory chronic condition that affects the skin of children and adults and has an important impact on the quality of life. Treatments for AD are based on environmental controls, topical and systemic therapies, and allergen-specific immunotherapy (AIT). However, it remains unclear the effectiveness and adverse events of AIT and all conventional topical treatments compared with placebo and each other for AD.

Methods: We will search five electronic databases [Central Cochrane register of controlled trials (CENTRAL), MEDL INE, EMBASE, CINAHL, and LILACS] from inception until November 2019 with no language restriction, and we will include experimental studies [randomized controlled trials (RCTs), and quasi-RCTs]. The primary outcome is global and specific skin symptoms assessment. Secondary outcomes are hospital length of stay, quality of life, and adverse events. Reviewers independently will extract data from the studies that meet our inclusion criteria and will assess the risk of bias of individual primary studies. We will conduct random effects pairwise meta-analyses for the observed pairwise comparisons with at least two trials. Then, we will perform random-effects Bayesian network meta-analysis (NMA) to obtain treatment effects for all possible comparisons and to provide a hierarchy of all interventions for each outcome. Possible incoherence between direct and indirect sources of evidence will be investigated locally (if possible) and globally. To investigate sources of statistical heterogeneity, we will perform a series of meta-regression analyses based on pre-specified important effect modifiers. Two authors will appraise the certainty of the evidence for each outcome applying the GRADE's framework for NMA.

(Continued on next page)
\end{abstract}

\footnotetext{
* Correspondence: jj.yepesn@uniandes.edu.co

'School of Medicine, Universidad de los Andes, Bogotá D.C., Colombia

${ }^{5}$ Pulmonology Service, Internal Medicine Section, Fundación Santa Fe de

Bogotá University Hospital, Bogotá D.C., Colombia

Full list of author information is available at the end of the article
}

(c) The Author(s). 2020 Open Access This article is licensed under a Creative Commons Attribution 4.0 International License, which permits use, sharing, adaptation, distribution and reproduction in any medium or format, as long as you give appropriate credit to the original author(s) and the source, provide a link to the Creative Commons licence, and indicate if changes were made. The images or other third party material in this article are included in the article's Creative Commons licence, unless indicated otherwise in a credit line to the material. If material is not included in the article's Creative Commons licence and your intended use is not permitted by statutory regulation or exceeds the permitted use, you will need to obtain permission directly from the copyright holder. To view a copy of this licence, visit http://creativecommons.org/licenses/by/4.0/ The Creative Commons Public Domain Dedication waiver (http://creativecommons.org/publicdomain/zero/1.0/) applies to the data made available in this article, unless otherwise stated in a credit line to the data. 
(Continued from previous page)

Discussion: The findings of this systematic review will shed the light on the effectiveness and adverse events of all possible comparisons for treating $\mathrm{AD}$ and on the quality of the collated evidence for recommendations. It will also provide critical information to health care professionals to comprehend and manage this disease at different age stages, treatment type, duration, and severity of atopic dermatitis.

\section{Systematic review registration: PROSPERO Protocol ID CRD42019147106}

Keywords: Atopic dermatitis, Topical administration, Immunotherapy, Systematic review, Meta-analysis, Network meta-analysis, GRADE approach

\section{Background}

Atopic dermatitis (AD) is an inflammatory chronic condition of the skin that affects 15 to $20 \%$ of children and 3 to $5 \%$ of adults worldwide [1-3]. AD prevalence currently seems to be increasing in Africa, East Asia, and some parts of Europe [4]. Its increasing prevalence leads to direct US national costs of $\$ 3.8$ billion dollars per year [5]. The main clinical features of AD are dry skin, eczema, and pruritus [2]. Pruritic erythematous papulevesicles characterize acute eczema [6,7]. Subacute and chronic lesions usually correspond to drier and desquamative lesions with excoriations, lichenification, and fissures areas due to chronic skin scraping $[2,6]$. AD has an important impact on the quality of life, as it affects emotional health and socialization, especially when lesions are visible, and symptoms are not controlled $[8,9]$.

Therapy for AD focus on symptoms improvement, avoidance of exacerbations, and minimizing therapeutic risk [10]. Treatments are based on environmental measures, topical and systemic treatments, and allergenspecific immunotherapy (AIT) $[6,11-15]$. Conventional topical treatments such as skin moisturizers and emollients are the first-line treatments due to their effect in maintaining skin hydration [16]. Corticosteroids, calcineurin inhibitors, janus-kinase (JAK) inhibitors, and phosphodiesterase-4 (PDE-4) inhibitors in their topical presentations are recommended in cases of noncontrolled AD [6, 12-15, 17]. Further, in severe forms of $\mathrm{AD}$ with treatment failure with topical treatment, it is necessary to start a systemic therapy [6]. However, despite all these available treatments, a subgroup of patients remains clinically uncontrolled [10]. These patients have poor quality of life and they are frequently seeking medical attention without achieving adequate control of their symptoms [18].

AIT, an intervention that involves the administration of increasing amounts of a specific allergen to an allergic patient, has demonstrated promising results to decrease the severity of the disease in patients with $\mathrm{AD}$ that do not require systemic treatment $[19,20]$. Since published evidence shows that AIT modifies the immune response to aeroallergens in patients with allergic rhinitis and asthma, AIT might modify the immune response in patients with $\mathrm{AD}$ improving their cutaneous symptoms [21]. However, the efficacy and adverse events of AIT for $\mathrm{AD}$ have not been directly or indirectly compared with all conventional topical treatments. Therefore, we aim to compare the effectiveness and adverse events of AIT versus conventional topical treatments or placebo in patients with $\mathrm{AD}$ through a systematic review with NMA.

\section{Methods}

This protocol for a systematic review with NMA is registered in PROSPERO (CRD42019147106), an international database of prospectively registered systematic reviews in health, and it will be conducted in accordance to the Cochrane Handbook [22]. For the development of the present protocol, we used to the Preferred Reporting Items for Systematic review and Meta-Analysis Protocols (PRISMA-P) [23] (see Additional file 1).

\section{Data source and search strategy}

We will search MEDLINE, EMBASE, CINAHL, LILACS, and the Cochrane Controlled Register of Trials (CENT RAL) databases for relevant literature from inception until November 2019. A librarian from the Department of Health Research Methods, Evidence, and Impact (HEI) from McMaster University with expertise in designing search strategies for systematic reviews created our search strategy (see Additional file 2). Search will not be restricted to any language, stage, or date of publication. We will also conduct a manual search of RCTs and grey literature through clinicaltrials.gov and the WHO international registry of clinical trials, and summary of conferences, and dissertation databases through ProQuest Dissertations and thesis database. We will contact the authors of non-published works to guarantee eligibility.

\section{Eligibility criteria \\ Population}

Adults or children with mild, moderate, or severe AD, as defined by the investigators, with or without allergic sensitization to an inhalant or food. All countries and settings are eligible for inclusion. 


\section{Interventions and comparisons}

Topical corticosteroids, topical calcineurin inhibitors, topical PDE-4 inhibitors, topical JAK inhibitors, coal tar, topical aryl hydrocarbon receptor activators, subcutaneous AIT or sublingual AIT for any type of allergen, placebo, or standard care. We define standard care as it is defined by the investigators, including skin hydration and moisturization, and bathing with soaps and washes, among others. In the case of co-interventions, we will explore whether the intervention effect is modified by the addition of supplementary intervention, such as the presence of standard care, through subgroup analysis. Comparisons may include individual and/or combined interventions at any dose or presentation.

\section{Outcomes}

The primary outcomes include (1) The proportion of patients (or parents) that inform or present a global improvement of cutaneous symptoms at the end of treatment, and (2) the proportion of patients (or parents) that inform or present an improvement of specific symptoms such as erythema, vesicles, xerosis, excoriation, and/or lichenification of the skin at the end of treatment. Secondary outcomes include (3) preventing the development of asthma and/or other allergy diseases such allergic rhinitis and food allergy, (4) the severity of the disease at the end of treatment defined by scores (SCORAD, EASI, or any other used in the study) that assess lesion intensity and/or extension, symptoms, disease course, and epidermal function, (5) changes in the quality of life in both mental and physical health, and (6) local or systemic adverse events.

We will investigate these outcomes by short term $(\leq$ 16 weeks of treatment), and long-term, ( $>16$ weeks of treatment. We will use data at the end of the study in case of multiple times measurements. Multiple times will also be classified as short term and long term based on data at the end of the study.

\section{Study designs}

Experimental studies [randomized clinical trials (RCTs) and quasi-RCTs]. We will not limit study inclusion by publication status, the language of dissemination, duration of follow-up, or period of study conduct.

\section{Study selection}

Prior to article selection we will conduct a calibration process to determine the agreement between the reviewers assessing the kappa statistic expecting to get a score greater than 0.7 [22]. Reviewers will go through the search hits by reading titles and abstracts and evaluate its eligibility in an independent and duplicate approach. For each potentially relevant study, we will obtain the full text and will assess its inclusion. In case of disagreement between reviewers, a third author will review the study and resolve its inclusion. Literature search results will be uploaded to Covidence ${ }^{\circ}$ Software, an Internet-based software program that facilitates collaboration among reviewers during the study selection process.

\section{Data extraction}

We will extract the data, in duplicate, from the eligible studies using a pre-specified Microsoft Excel $^{\circ}$ form. The following data will be extracted: characteristics of the study (design, year, follow-up duration, sample size per arm, environment, and country), patient characteristics (average age, in- or out-patient setting, duration of the disease since first diagnosis, allergenic sensitization), and intervention characteristics (doses, routes of administration). For binary outcomes, we will extract the number of events, number of missing participant outcome data, and number of randomized participants for each arm of every trial, whereas for continuous outcomes, we will extract the mean, standard deviation, number of missing participant outcome data and number of randomized participants for each arm of every trial. To tackle wellknown challenges with the data extraction for continuous outcomes, we will consider the directions described in Section 7.7.3 of the Cochrane Handbook [22]; for instance, to obtain missing standard deviations from reported standard errors, confidence intervals, $t$ values, or $p$ values for the difference in means. All reviewers will assess the data extraction checklist before the extraction. Five reviewers (LG, AV, HM, AG, and JY) will extract data independently and by duplicate. Any disagreement will be assessed by a third reviewer (EG or JY).

\section{Assessment of risk of bias in included studies}

We will use a modify version of the Cochrane's Risk of Bias (RoB) tool to assess the RoB in the included studies [24]. In duplicate and independently, we will assess the following domains: sequence for random allocation, allocation concealment, blinding of participants and personnel, blinding of outcome assessment, incomplete outcome data, selective reporting, and other biases. Each domain will be assessed as "definitively yes," "probably yes," "probably no," or "definitively no." Any disagreement will be assessed by a third reviewer (EC or JY).

\section{Random-effects pairwise meta-analysis}

Clinical and methodological heterogeneity is expected between studies (issue addressed in the section: certainty evaluation in network metanalysis effect estimation) which may lead to statistical heterogeneity. To encompass statistical heterogeneity, random-effects (RE) metaanalysis will be performed for each direct comparison that is informed by at least two trials. Compared to 
fixed-effect model, RE model [25] inherently leads to wider confidence/credible intervals around the pooled treatment effect depending on the extent of betweentrial variance as measured by the parameter $\tau^{2}$. Under the RE model, the observed treatment effect in a study is a function of the average treatment effect from all studies, the random-effect and the sampling error of that study [26]. In other words, RE model assumes that the true treatment effects of the included trials are randomly sampled from a specific distribution (commonly a normal distribution). Binary outcomes will be analyzed using the odds ratio (OR), whereas continuous outcomes using the mean difference if all trials have used the same scale or standardized mean difference (SMD) when scales are different. To avoid replacing zero cells with abstract thresholds to estimate OR and its variance, we will apply Bayesian RE meta-analysis with binary likelihood and logit link to model the binary outcome data [27]. For continuous outcomes, we will apply Bayesian RE meta-analysis with normal likelihood and identity link [27]. Results will be reported using the posterior mean for the treatment effects but the posterior median for $\tau^{2}$ alongside their $95 \%$ credible intervals. To account for possible missing participant outcome data in the included trials, we will apply the pattern-mixture model with informative missingness odds ratio parameter as proposed by Turner et al. [28] for binary outcomes, and the pattern-mixture model with informative missingness difference of means parameter as proposed by Mavridis et al. [29] for continuous outcomes. Both missingness parameters will be modeled under the missing at random assumption with an independent, uncorrelated structure for each arm of every trial.

\section{Random-effects network meta-analysis}

We expect that many of the available treatments to treat $\mathrm{AD}$ have not been compared in any trial. In the absence of direct evidence for a comparison, indirect evidence can be obtained by combining trials that compare the interventions with a common comparator. By incorporating direct and indirect evidence in a single analysis, NMA provides an internally coherent set of relative treatment effects for all possible comparisons and, therefore, allows for a formal hierarchy of the interventions from the best to worst for each outcome [30]. If transitivity assumption (i.e., similarity of included trials in terms of clinical and methodological characteristics that comprise important effect-modifiers) is deemed plausible, NMA can be safely applied to provide credible results [31]. Otherwise, violation of transitivity assumption may cause incoherence between direct and one or more indirect effects beyond between-trial variance, and thus, reduce our certainty to the NMA results [31]. We will assess the plausibility of transitivity assumption by investigating the distribution of important effect modifiers in each observed comparison [32]. We will apply Bayesian RE NMA with consistency equation and incorporation of multi-arms trials to accommodate the anticipated statistically heterogeneity and to account for the correlation between treatment effects that share the same control arm in multi-arm trials [27, 33]. Betweentrial variance will be assumed common in the whole network to enable estimation of the parameter for comparisons with few trials, as information is "borrowed" by comparisons with many trials [31]. Under this assumption, the correlation between treatment effects in multiarm trials equals 0.5 [27]. Coherence, the statistical manifestation of transitivity, will be investigated locally and globally. For the former, we will apply the nodesplitting approach $[34,35]$ using the R-package gemtc [36] to automatically identify the comparisons to split in closed loops of interventions, whereas for the latter, we will compare the model-fit and complexity of the NMA model with and without consistency equation using the deviance information criterion (DIC) which provides a measure of model fit penalized for model complexity [37]. The model with lower DIC will be considered to have a better compromise between model fit and complexity [34, 38].

To illustrate the network geometry, we will create network plots for each outcome. The plots will display visual information of the evidence retrieved for each outcome regarding the number of trials and patients involved in each direct comparison.

In line with the statistical analysis for pairwise metaanalysis, we will use OR and mean difference or SMD as effect measures for binary and continuous outcomes respectively, and we will report the posterior mean for the treatment effects but the posterior median for $\tau^{2}$ alongside their $95 \%$ credible intervals. Furthermore, we will extent the pattern-mixture model of Turner et al. [28] for binary missing participant outcome data to operate in a network of interventions [39], and we use the pattern-mixture model of Mavridis et al. [29] to incorporate continuous missing participant outcome data as observed in the analyzed networks. We will create league tables for each outcome to present the NMA results for all possible comparisons as well as the results from pairwise meta-analysis for the observed comparisons with at least two trials. Furthermore, for each outcome, we will create forest-plots to present the NMA treatment effects alongside the respective direct and indirect treatment effects of comparisons with the reference intervention of the network. We will also present several measures of intervention hierarchy including the rank probabilities, ranks, and surface under the cumulative ranking curve (SUCRA) values [40]. Specifically, we will create rankograms for each intervention and outcome to fully 
illustrate the uncertainty across the ranks [40]. We will also present SUCRA plots to illustrate the cumulative ranking probabilities for each intervention and outcome [40]; for each intervention, SUCRA value indicates the percentage of effectiveness (or safety) of that intervention as compared to an imaginary intervention that is always the best with certainty [41]. For each outcome, the best treatment will have high SUCRA value and the worst treatment low SUCRA value. To aid the interpretation of the results in terms of hierarchy and relative treatment effects, we will incorporate the posterior median ranks and posterior mean SUCRA values alongside their $95 \%$ credible interval in the aforementioned forestplots. We will create a scatter diagram to identify the best balance between efficacy and safety.

\section{Investigating statistical heterogeneity}

Thirteen a priori important effect-modifiers will be considered to investigate possible incoherence and statistical heterogeneity. Possible effect-modifiers are (1) severity of the disease: mild/moderate vs severe (based on SCORAD scale); (2) allergy sensitization proven by positive skin prick test or circulating levels of allergen-specific IgE antibody or total IgE detected vs no allergy sensitization proven; (3) pediatric vs adult population (18 years or older); (4) route of allergenic immunotherapy administration: sublingual vs subcutaneous; (5) duration of treatment: short term (less than 16 weeks) vs long term (more than 16 weeks); (6) type of allergen: dust mites vs pollen vs pet allergen; (7) adverse events: local vs systemic; (8) small-study effects; (9) RoB level of the trials included: low vs high risk of bias; (10) funding resource (pharmaceutical companies, grants or other financial resource); (11) treatment type: topical corticosteroids, calcineurin inhibitors, topical PDE-4 inhibitors, JAK topical inhibitors, sublingual allergen immunotherapy, subdermal allergen immunotherapy; (12) same standard care intervention is delivered to both the intervention and comparator groups; and (13) presence of other allergic diseases such as asthma, allergic rhinitis, and food allergy. We anticipate a stronger treatment effect in patients with severe $A D$, subcutaneous AIT, patients with allergy sensitization proved, duration of more than 16 weeks, in smaller trials, and patients with other allergic diseases. In case of bias, RCTs with a higher RoB may show bigger treatment effects than RCTs with lower RoB. The impact of the effect-modifiers on the NMA results will be investigated by applying Bayesian meta-regression RE models and assuming exchangeable regression coefficients [42].

\section{Assessment of small-study effects and possible publication bias}

Since different studies investigate different comparisons, we will provide a comparison-adjusted funnel plot to investigate graphically the presence of possible smallstudy effects [22, 43]. This plot is an extension of the funnel plot used in pairwise meta-analysis, as it distinguishes among the different trial-specific treatment effects for different comparisons [43]. We will apply three comparison-adjusted funnel plots where studies will be labeled as (i) active- versus placebo-controlled trials, (ii) old versus new intervention, and (iii) sponsored versus non-sponsored intervention. In the presence of funnel plot asymmetry, we will use selection model to investigate the possibility of publication bias [44].

\section{Model specification in pairwise and network meta- analysis}

In both pairwise and network meta-analysis, prior normal distributions centered at 0 with variance equal 10 , 000 will be used for all location parameters of the models, whereas for the parameter $\tau^{2}$ we will use proper empirical priors tailored to the intervention-comparison type and the investigated outcome as suggested by Turner el al [45]. for binary outcomes and Rhodes et al. [46] for continuous outcomes. For the meta-regression analyses, prior normal distribution centered on 0 with variance equal 10,000 and uniform distribution over the interval $[0,5]$ will be assigned on the common mean and standard deviation of the normally distributed regression coefficients, respectively. For all Bayesian analyses, we will apply three parallel chains with different initial values using 200,000 updates and a burn-in of 20, 000 MCMC samples. We will assess convergence using the Gelman-Rubin convergence diagnostic, $\hat{R}$, and through inspection of trace and autocorrelation plots [47]. All analyses will be performed in JAGS [48] using the R-package gemtcs [36] and R2jags [49].

\section{Classifying certainty of effect estimates in NMA}

The reviewers will evaluate in pairs and independently the certainty of estimations (quality of evidence) for each informed outcome according to GRADE [50]. Certainty will be classified in four levels: high, moderate, low, and very low. We will evaluate and classify each direct comparison result according to the following categories: RoB [51], inconsistency (determined by heterogeneity as previously mentioned) [52], indirectness [53], and publication bias [54]. We will also assess imprecision on the credible intervals around the network estimates according to the GRADE for NMA updated guidance [27].

To evaluate the certainty of the NMA estimations, we will follow four steps: (1) present the direct and indirect estimates of effect for the pairwise comparison, (2) rate the certainty of both of these estimates, (3) present the network estimate for the pairwise comparison, and (4) rate the certainty of the network estimate, based on the 
ratings of the direct and indirect estimates and the assessment of coherence. For rating the certainty of the indirect estimates, we will focus their assessment on the most-dominant first-order loop [26]. We will assess the certainty of indirect effect estimates if the certainty of the direct estimates is not high, and the contribution of the direct evidence to the network estimate is lower as that of the indirect evidence [27].

Our judgment of certainty in the NMA estimation to any paired comparison will be the highest of the certainty qualifications between the direct and indirect comparisons that contribute to the model. Nonetheless, we can reduce the certainty in the network estimation if we find that direct and indirect estimations are inconsistent and/or imprecise. Using the updated GRADE approach we will also assess incoherence or inconsistency, which is defined as the effect difference between direct and indirect estimations [55].

\section{Discussion}

To our knowledge, this protocol describes the first systematic review and network meta-analyses that specifically examines the effectiveness and adverse events of topical and allergen immunotherapy for atopic dermatitis. Previous reviews have described the efficacy of allergen-specific immunotherapy for atopic dermatitis [56], the efficacy of subcutaneous and sublingual grass allergen immunotherapy [57], and a Cochrane review about specific allergen immunotherapy for the management of atopic dermatitis [58]. This systematic review will describe the proportion of patients with global improvement of cutaneous symptoms, proportion of patients that present improvement in specific symptoms, the severity of the disease at the end of treatment, changes in quality of life, and adverse events across multiple interventions. Our NMA will allow the comparison and ranking of treatments that have not been compared head to head.

Our target users are allergy and dermatology practitioners, as well as researchers and healthcare policymakers. We plan to present our results at national and international meetings.

Limitations to the review may include the diverse outcome measurements and differences in trial design that may limit our capacity to combine results from different clinical trials. Additionally, limited data in certain interventions may limit the ability to run an NMA on all outcomes described.

The findings from this systematic review and NMA will help health care professionals to make evidencebased decisions for AD patients in the absence of head to head trial comparisons in topical and AIT interventions. Our findings will also identify evidence gaps and decrease uncertainty in relative and absolute estimates of the interventions being compared.

\section{Supplementary information}

Supplementary information accompanies this paper at https://doi.org/10. 1186/s13643-020-01472-w.

Additional file 1. PRISMA-P 2015 Checklist.

Additional file 2. Medline Search Strategy.

\section{Abbreviations}

AD: Atopic dermatitis; AIT: Allergen-specific immunotherapy; GRADE: Grading of Recommendation, Assessment, Development and Evaluation; JAK: Janus kinase; NMA: Network meta-analysis; OR: Odds ratio; PDE-

4: Phosphodiesterase-4; RCT: Randomized clinical trials; RoB: Risk of bias; SMD: Standard mean difference; SUCRA: Surface under the cumulative ranking curve

\section{Authors' contributions}

$J J Y, L G G, H J M$, and AV conceived the review. All authors designed the protocol. LGG and HJM conducted the preliminary searches. JJY is the guarantor of the review. All authors reviewed the manuscript and read and approved the final manuscript.

\section{Funding}

This study is supported by a research grant from the Faculty of Medicine, University of Los Andes and the University Hospital Fundación Santa Fe de Bogotá.

\section{Availability of data and materials}

The datasets created and analyzed during this review will be available from the corresponding author upon reasonable request.

\section{Ethics approval and consent to participate}

Not applicable.

Consent for publication

Not applicable.

\section{Competing interests}

The authors declare no competing interests.

\section{Author details}

${ }^{1}$ School of Medicine, Universidad de los Andes, Bogotá D.C., Colombia. ${ }^{2}$ Midwifery Research and Education Unit, Hannover Medical School,

Hannover, Germany. ${ }^{3}$ Dermatology Section, University of Antioquia, Medellín, Colombia. ${ }^{4}$ Department of Health Research Methods, McMaster University, Hamilton, Ontario, Canada. ${ }^{5}$ Pulmonology Service, Internal Medicine Section, Fundación Santa Fe de Bogotá University Hospital, Bogotá D.C., Colombia.

Received: 10 March 2020 Accepted: 2 September 2020

Published online: 28 September 2020

\section{References}

1. Asher MI, Montefort S, Björkstén B, Lai CK, Strachan DP, Weiland SK, et al. Worldwide time trends in the prevalence of symptoms of asthma, allergic rhinoconjunctivitis, and eczema in childhood: ISAAC Phases One and Three repeat multicountry cross-sectional surveys. Lancet. 2006;368(9537):733-43.

2. Eichenfield LF, Tom WL, Chamlin SL, Feldman SR, Hanifin JM, Simpson EL, et al. Guidelines of care for the management of atopic dermatitis: Section 1. Diagnosis and assessment of atopic dermatitis Work Group. J Am Acad Dermatol. 2014;70(2):338-51.

3. Barbarot S, Auziere S, Gadkari A, Girolomoni G, Puig L, Simpson EL, et al. Epidemiology of atopic dermatitis in adults: Results from an international survey. Allergy Eur J Allergy Clin Immunol. 2018;73(6):1284-93.

4. Deckers IAG, McLean S, Linssen S, Mommers M, van Schayck CP, Sheikh A. Investigating international time trends in the incidence and prevalence of atopic eczema 1990-2010: A systematic review of epidemiological studies. PLOS ONE. 2012;7.

5. Mancini AJ, Kaulback K, Chamlin SL. The socioeconomic impact of atopic dermatitis in the United States: A systematic review. Pediatr Dermatol. 2008; 25:1-6. 
6. Simpson, E. L, Leung D.Y.M, Eichenfield LF BM. Atopic Dermatitis in Fitzpatrick's Dermatology. 9th ed. Kang S, Amagai M, Bruckner AL, Enk AH, Margolis DJ, McMichael AJ, et al. editor. New York: McGraw-Hill Education; 2019.

7. Leung DYM. Clinical implications of new mechanistic insights into atopic dermatitis. Vol. 28, Current Opinion in Pediatrics: Lippincott Williams and Wilkins; 2016. p. 456-62.

8. Patel KR, Immaneni S, Singam V, Rastogi S, Silverberg J. Association between atopic dermatitis, depression, and suicidal ideation: A systematic review and meta-analysis. J Am Acad Dermatol. 2019;80(2):402-10.

9. Sanclemente G, Burgos C, Nova J, Hernández F, González C, Reyes MI, et al. El impacto de las enfermedades cutáneas en la calidad de vida: Un estudio multicéntrico. Actas Dermosifiliogr. 2017;108(3):244-52.

10. Schneider L, Tilles S, Lio P, Boguniewicz M, Beck L, Lebovidge J, et al. Atopic dermatitis: A practice parameter update 2012. J Allergy Clin Immunol. 2013; 131(2).

11. Harper J, Giehl K, Bingham EA. Guidelines to Management of Atopic Dermatitis. In: Harper's Textbook of Pediatric Dermatology. Oxford, UK Wiley-Blackwell; 2011. p. 30.1-30.14

12. Wollenberg A, Barbarot S, Bieber T, Christen-Zaech S, Deleuran M, FinkWagner A, et al. Consensus-based European guidelines for treatment of atopic eczema (atopic dermatitis) in adults and children: part I. J Eur Acad Dermatology Venereol. 2018;32(5):657-82.

13. Wollenberg A, Barbarot S, Bieber T, Christen-Zaech S, Deleuran M, FinkWagner A, et al. Consensus-based European guidelines for treatment of atopic eczema (atopic dermatitis) in adults and children: part II. J Eur Acad Dermatology Venereol. 2018;32(6):850-78.

14. Williams HC, Jburney PG, Pembroke AC, Hay RJ. The U.K. Working Party's Diagnostic Criteria for Atopic Dermatitis. III. Independent hospital validation. Br J Dermatol. 1994;131(3):406-16.

15. Eichenfield LF, Tom WL, Berger TG, Krol A, Paller AS, Schwarzenberger K, et al. Guidelines of care for the management of atopic dermatitis: Section 2. Management and treatment of atopic dermatitis with topical therapies. J Am Acad Dermatol. 2014;71(1):116-32.

16. Chamlin SL, Kao J, Frieden IJ, Sheu MY, Fowler AJ, Fluhr JW, et al. Ceramidedominant barrier repair lipids alleviate childhood atopic dermatitis: Changes in barrier function provide a sensitive indicator of disease activity. J Am Acad Dermatol. 2002;47(2):198-208.

17. Sidbury R, Davis DM, Cohen DE, Cordoro KM, Berger TG, Bergman JN, et al. Guidelines of care for the management of atopic dermatitis: Section 3. Management and treatment with phototherapy and systemic agents. J Am Acad Dermatol. 2014;71(2):327-49.

18. Wittkowski A, Richards HL, Griffiths CEM, Main CJ. The impact of psychological and clinical factors on quality of life in individuals with atopic dermatitis. J Psychosom Res. 2004;57(2):195-200.

19. Cox L, Nelson H, Lockey R, Calabria C, Chacko T, Finegold I, et al. Allergen immunotherapy: A practice parameter third update. J Allergy Clin Immunol. 2011;127(1 SUPPL)

20. Burks AW, Calderon MA, Casale T, Cox L, Demoly P, Jutel M, et al. Update on allergy immunotherapy: American Academy of Allergy, Asthma \& Immunology/European Academy of Allergy and Clinical Immunology/ PRACTALL consensus report. J Allergy Clin Immunol. 2013;131(5).

21. Gendelman SR, Lang DM. Specific immunotherapy in the treatment of atopic dermatitis: A systematic review using the GRADE system. Ann Allergy Asthma Immunol. 2013;111(6):555-61.

22. Higgins JPTGS, editor. Cochrane Handbook for Systematic Reviews of Interventions Version 5.1.0: The Cochrane Collaboration; 2011.

23. Shamseer L, Moher D, Clarke M, Ghersi D, Liberati A, Petticrew M, et al. Preferred reporting items for systematic review and meta-analysis protocols (prisma-p) 2015: Elaboration and explanation. BMJ. 2015;349(January):1-25.

24. Akl EA, Sun X, Busse JW, Johnston BC, Briel M, Mulla S, et al. Specific instructions for estimating unclearly reported blinding status in randomized trials were reliable and valid. J Clin Epidemiol. 2012;65(3):262-7.

25. Deeks, Jonathan J, Higgins J.P.T AD. Chapter 9: Analysing data and undertaking. Cochrane Handbook for Systematic Reviews of Interventions Version 5.1.0 (updated March 2011). 2011.

26. Borenstein $M$, Hedges $L$ V., Higgins JPT, Rothstein HR. A basic introduction to fixed-effect and random-effects models for meta-analysis. Res Synth Methods. 2010 [cited 2014 Feb 24];1(2):97-111. Available from: http://doi. wiley.com/10.1002/jrsm.12.

27. Dias S, Sutton AJ, Ades A, Welton NJ. Evidence synthesis for decision making 2: a generalized linear modeling framework for pairwise and network meta-analysis of randomized controlled trials. Med Decis Mak. 2013;33(5):607-17.

28. Turner NL, Dias S, Ades AE, Welton NJ. A Bayesian framework to account for uncertainty due to missing binary outcome data in pairwise meta-analysis. Stat Med. 2015;34(12):2062-80.

29. Mavridis D, White I, Higgins J, Cipriani A, Salanti G. Allowing for uncertainty due to missing continuous outcome data in pairwise and network metaanalysis. Stat Med. 2015;34(5):721-41.

30. Caldwell DM. An overview of conducting systematic reviews with network meta-analysis. Syst Rev. 2014;3(1):1-4.

31. Salanti G. Indirect and mixed-treatment comparison, network, or multipletreatments meta-analysis: many names, many benefits, many concerns for the next generation evidence synthesis tool. Res Synth Methods. 2012;3(2): 80-97 Available from: http://doi.wiley.com/10.1002/jrsm.1037.

32. Jansen JP, Naci $H$. Is network meta-analysis as valid as standard pairwise meta-analysis? It all depends on the distribution of effect modifiers. BMC Med. 2013:11(1).

33. Franchini AJ, Dias S, Ades AE, Jansen JP, Welton NJ. Accounting for correlation in network meta-analysis with multi-arm trials. Res Synth Methods. 2012;3(2):142-60 Available from: http://doi.wiley.com/10.1002/ jrsm.1049.

34. Dias S, Welton N, Caldwell D, Ades A. Checking consistency in mixed treatment comparison meta-analysis. Stat Med. 2010;29(7-8):932-44.

35. van Valkenhoef G, Dias S, Ades AE, Welton NJ. Automated generation of node-splitting models for assessment of inconsistency in network metaanalysis. Res Synth Methods. 2016;7(1):80-93.

36. van Valkenhoef GKJ. gemtc: Network meta-analysis using Bayesian methods; 2016

37. Spiegelhalter DJ, Best NG, Carlin BP, van der Linde A. Bayesian Measures of Model Complexity anf Fit. J R Stat Soc Ser B (Stat Methodol). 2002;64(4): 583-639.

38. Dias S, Welton NJ, Sutton AJ, Caldwell DM, Lu G, Ades AE. Evidence synthesis for decision making 4: Inconsistency in networks of evidence based on randomized controlled trials. Med Decis Mak. 2013;33(5):641-56.

39. Spineli LM. An empirical comparison of Bayesian modelling strategies for missing binary outcome data in network meta-analysis. BMC Med Res Methodol. 2019;19(1):86 Available from: https://bmcmedresmethodol. biomedcentral.com/articles/10.1186/s12874-019-0731-y.

40. Salanti G, Ades AE, loannidis JPA. Graphical methods and numerical summaries for presenting results from multiple-treatment meta-analysis: an overview and tutorial. J Clin Epidemiol. 2011;64(2):163-71. Available from. https://doi.org/10.1016/j.jclinepi.2010.03.016.

41. Mavridis D, Giannatsi M, Cipriani A, Salanti G. A primer on network metaanalysis with emphasis on mental health. Evid Based Ment Health. 2015; 18(2):40-6.

42. Cooper NJ, Sutton AJ, Morris D, Ades A, Welton NJ. Addressing betweenstudy heterogeneity and inconsistency in mixed treatment comparisons: Application to stroke prevention treatments in individuals with nonrheumatic atrial fibrillation. Stat Med. 2009:28:1861-81.

43. Chaimani A, Salanti G. Using network meta-analysis to evaluate the existence of small-study effects in a network of interventions. Res Synth Methods. 2012;3(2):161-76 Available from: http://doi.wiley.com/10.1002/ jrsm.57.

44. Mavridis D, Welton NJ, Sutton A, Salanti G. A selection model for accounting for publication bias in a full network meta-analysis. Stat Med. 2014;33(30):5399-412.

45. Turner RM, Davey J, Clarke MJ, Thompson SG, Higgins JP. Predicting the extent of heterogeneity in meta-analysis, using empirical data from the Cochrane Database of Systematic Reviews. Int J Epidemiol. 2012;41(3):818-27.

46. Rhodes KM, Turner RM, Higgins JPT. Predictive distributions were developed for the extent of heterogeneity in meta-analyses of continuous outcome data. J Clin Epidemiol. 2015;68(1):52-60. Available from. https://doi.org/10. 1016/j.jclinepi.2014.08.012.

47. Gelman A, Rubin DB. Inference from iterative simulation using multiple sequences. Stat Sci. 1992.

48. Plummer M. JAGS Version 3.4 .0 user manual; 2013. p. 1-42.

49. Su Y, Yajima M. R2jags: Using R to Run "JAGS". 2015. Available from: https:// cran.r-project.org/package $=$ R2jags.

50. Guyatt G, Oxman AD, Akl EA, Kunz R, Vist G, Brozek J, et al. GRADE guidelines: 1. Introduction - GRADE evidence profiles and summary of findings tables. J Clin Epidemiol. 2011;64(4):383-94. 
51. Guyatt GH, Oxman AD, Vist G, Kunz R, Brozek J, Alonso-Coello P, et al. GRADE guidelines: 4. Rating the quality of evidence - Study limitations (risk of bias). J Clin Epidemiol. 2011;64(4):407-15.

52. Guyatt GH, Oxman AD, Kunz R, Woodcock J, Brozek J, Helfand M, et al. GRADE guidelines: 7. Rating the quality of evidence - Inconsistency. J Clin Epidemiol. 2011;64(12):1294-302.

53. Guyatt GH, Oxman AD, Kunz R, Woodcock J, Brozek J, Helfand M, et al. GRADE guidelines: 8. Rating the quality of evidence - Indirectness. J Clin Epidemiol. 2011;64(12):1303-10

54. Guyatt GH, Oxman AD, Montori V, Vist G, Kunz R, Brozek J, et al. GRADE guidelines: 5. Rating the quality of evidence - Publication bias. J Clin Epidemiol. 2011;64(12):1277-82

55. Brignardello-Petersen R, Bonner A, Alexander PE, Siemieniuk RA, Furukawa TA, Rochwerg B, et al. Advances in the GRADE approach to rate the certainty in estimates from a network meta-analysis. J Clin Epidemiol. 2018; 93:36-44.

56. Bae JM, Choi YY, Park CO, Chung KY, Lee KH. Efficacy of allergen-specific immunotherapy for atopic dermatitis: a systematic review and meta-analysis of randomized controlled trials. J Allergy Clin Immunol. 2013;132(1):110-7.

57. Nelson H, Cartier S, Allen-Ramey F, Lawton S, Calderon MA. Network metaanalysis shows commercialized subcutaneous and sublingual grass products have comparable efficacy. J Allergy Clin Immunol Pr. 2015;3(2):256-266.e3. Available from. https://doi.org/10.1016/j.jaip.2014.09.018.

58. Tam H, Calderon MA, Manikam L, Nankervis H, García Núñez I, Williams HC, et al. Specific allergen immunotherapy for the treatment of atopic eczema. Vol. 2016, Cochrane Database of Systematic Reviews: John Wiley and Sons Ltd; 2016.

\section{Publisher's Note}

Springer Nature remains neutral with regard to jurisdictional claims in published maps and institutional affiliations.

Ready to submit your research? Choose BMC and benefit from:

- fast, convenient online submission

- thorough peer review by experienced researchers in your field

- rapid publication on acceptance

- support for research data, including large and complex data types

- gold Open Access which fosters wider collaboration and increased citations

- maximum visibility for your research: over $100 \mathrm{M}$ website views per year

At $\mathrm{BMC}$, research is always in progress.

Learn more biomedcentral.com/submissions 